\title{
Beeinflusst die medizinische Versorgungsstruktur den Anteil Todesfälle im Spital?
}

\author{
Eine ökologische Analyse in den MS-Regionen der Schweiz
}

Summary

Does the structure of medical services affect the proportion of deaths in hospitals? An ecological analysis in the mobility regions of Switzerland

Objectives: With the discussion about measures to reduce over-capacities in the health services in mind, we examined the influence of medical supply structures and services on hospital utilisation directly before death.

Methods: Based on the specification of the place of dying from the death certificates, we determined the proportions of deaths in hospitals in an ecological study. We analysed deaths of persons above age 65 in each of the 106 mobility regions of Switzeriand in the years 1979 to 1980 .

Results: The proportion of deaths occurring in hospitals varied between $27 \%$ and $81 \%$. Despite missing data regarding stathonary and ambulatory care semices, more than half of the variance could be explained by means of a multivariate regression anajysis. our results imply an inverse relation between the proportion of deaths in the hospital and the number of consultations provided by primary care physicians, as well as the number of nursing home beds. Further we observe a direct relation to the number of hospital beds in a region. All indicators are calculated per inhabitant.

Conclusions: In heath care supply planning, such systematic associations should be taken into account. We recommend to analyse regulaty interregional differences in supply and outcome of medical performances with the best avallable data.

Key-Words: Health services - Health services utilisation - Regional health planning Health services for the aged - Switzerland.
In der Schweiz sind unterschiedliche Massnahmen zum Abbau von Überkapazitäten im Gesundheitswesen Gegenstand politischer Debatten. Dabei wird u. a. auch eine übermässige und möglicherweise angebotsinduzierte Inanspruchnahme von Versorgungsstrukturen und Dienstleistungen diskutiert. Im Spitalbereich findet beispielsweise gegenwärtig ein Abbau des Angebotes statt. Solche gesundheitspolitischen Massnahmen können jedoch auch eine Unter- oder Fehlversorgung bestimmter Bevölkerungsgruppen oder eine Kostenverlagerung in andere Bereiche der medizinischen Versorgung zur Folge haben.

Für eine langfristig erfolgreiche Versorgungsplanung sind daher die Fragen nach der idealen Struktur und dem optimalen Ausmass des anzustrebenden Angebotes sowie nach den steuerbaren Einflussfaktoren auf die Inanspruchnahme mit ihren Auswirkungen auf das Gesundheits- und Gesellschaftssystem von zentraler Bedeutung. Zur Beantwortung dieser Fragen werden Informationen über Zusammenhänge zwischen Bedarf, Angebot und Inanspruchnahme der unterschiedlichen ambulanten und stationären Strukturen und Dienstleistungen benötigt ${ }^{1-5}$. Die unvollständige gesundheitsstatistische Datenlage erschwert die Durchführung entsprechender Untersuchungen ebenso wie die dezentrale und komplexe Organisation des schweizerischen Gesundheitssystems. In der Schweiz stehen regionale Informationen über Angebot und Inanspruchnahme von medizinischen Versorgungsstrukturen und Dienstleistungen nicht flächendeckend und nicht routinemässig zur Verfügung. Untersuchungen aus den USA zeigen jedoch bei MedicareBezügern einen Zusammenhang zwischen der Inanspruchnahme des Spitals (z.B. Hospitalisationsdauer) und dem Anteil der Todesfälle im Spital ${ }^{3,6}$.

Der Anteil der Todesfälle im Spital an den gesamten Todesfällen lässt sich aufgrund der Angaben zum Sterbeort in der Sterbefallstatistik des Bundesamtes für Statistik (BFS) 
auch für die Schweiz bestimmen. Aufgrund dieser Angaben können indirekte Hinweise über die Inanspruchnahme des Spitals gewonnen werden.

Dieser Inanspruchnahme unmittelbar vor dem Tode kommt insbesondere im Zusammenhang mit dem Anstieg der Gesundheitsausgaben mit zunehmender Nähe zum Todeszeitpunkt eine wichtige Bedeutung $\mathrm{zu}^{7,8}$ Andererseits besteht aus gesellschaftlicher Perspektive ein Widerspruch zwischen dem Wunsch der meisten älteren Menschen, bis zum Tode im eigenen Haushalt leben zu können ${ }^{9}$ und der Tatsache, dass ein grosser Anteil der Personen im Spital oder im Alters- und Pflegeheim verstirbt ${ }^{10}$.

Felder et al. ${ }^{7}$ zeigten für die Schweiz, dass $18 \%-22 \%$ der Gesundheitsausgaben von Rentnern im letzten Lebensjahr und davon $42 \%-49 \%$ im letzten Quartal vor dem Tode ausgegeben werden. Der grösste Anteil der gesamten Gesundheitsausgaben wird zudem für die stationäre Versorgung verwendet (1997: 47,1\%) ${ }^{11}$. Dabei ist die Mehrzahl der Spitalpatienten betagt oder hochbetagt ${ }^{12}$.

Die Anteile der Sterbefälle im Spital sind in den Industrieländern im entsprechenden Zeitraum weitgehend vergleichbar. In Untersuchungen aus Deutschland ${ }^{13}$, England ${ }^{14}$ und den USA ${ }^{15}$ lag der Anteil der Sterbefälle im Spital zwischen $51 \%$ und $56 \%$. Pritchard et al. ${ }^{6}$ zeigte zudem, dass diese Anteile in den einzelnen U.S. Hospital Referral Regions (HRRs) zwischen $23 \%$ und $54 \%$ variierten. In der Schweiz lag der Anteil der Todesfälle im Spital zwischen 1969 und 1986 konstant bei $55 \%{ }^{10}$, ohne signifikanten Unterschied zwischen Männern und Frauen. Im Zeitverlauf wurde bei Betagten ab 70-jährig eine Zunahme der Anteile von Sterbefällen im Spital festgestellt. In städtischen Verhältnissen waren diese Anteile zudem grösser als auf dem Lande (1986: $58 \%$ vs. $53 \%$ ).

Die Inanspruchnahme des Spitals wird in der internationalen Literatur meistens mit direkten Indikatoren beschrieben (z.B. Hospitalisationsdauer etc.). Einzelne Autoren verwendeten jedoch ausschliesslich oder in Ergänzung zu anderen Indikatoren auch den Sterbeort als Proxy-Variable ${ }^{3,6,16}$. Bei Pritchard et al. ${ }^{6}$ korrelierte der Anteil der Sterbefälle im Spital stark mit der Anzahl Spitaltage pro Medicare-Bezüger und Jahr. In den USA zeigte sich im Anschluss an die Änderung des Abrechnungssystems bei Medicare-Bezügern eine Verminderung der Anteile der Sterbefälle im Spital sowie eine vergleichbare Reduktion der Anzahl Spitaltage in den letzten 90 Tagen vor dem Tode ${ }^{3}$.

Bei den Einflussvariablen für die Inanspruchnahme des Spitals werden individuelle von regionalen Faktoren unterschieden. Als individuelle Einflussvariablen werden Krankheitszustand, informelle Pflegeunterstützung, Versicherungsdeckung sowie demographische, kulturelle, sozio- ökonomische Merkmale und Patientenwünsche diskutiert $^{2,10,17-22}$. Die regionalen Einflussvariablen beziehen sich entweder auf allgemeine Eigenschaften der Regionen (z.B. Demographie, Sprache, Kultur, Stadt-Land, Regionstyp etc.) oder aber auf Eigenschaften des regionalen medizinischen Versorgungssystems. In der mehrsprachigen und föderalistischen Schweiz kann beispielsweise der Sprachtyp als Indikator für einen kulturell-gesellschaftlichen oder (gesundheits-)politischen Einfluss auf das Inanspruchnahmeverhalten einer bestimmten Bevölkerung interpretiert werden. Dementsprechend zeigte Streckeisen ${ }^{10}$ für die Schweiz eine positive Assoziation zwischen dem Sterbeort Spital und den lateinischsprachigen Landesteilen. Sie diskutierte diese Beobachtung im Zusammenhang mit einer stärkeren Förderung der dezentralen und ambulanten Betreuungsmöglichkeiten sowie einem geringeren Ausbau der stationären Einrichtungen für Betagte in den lateinischsprachigen Landesteilen.

In der Literatur werden zahlreiche Merkmale des Gesundheitssystemes als mögliche Einflussfaktoren auf die Inanspruchnahme des Spitals diskutiert (z. B. Kapazität, Zugänglichkeit, Attraktivität der Versorgungsstrukturen, unterschiedliche Versicherungssysteme etc. $)^{2,36,616,17,21,22}$.

Pritchard et a1. ${ }^{6}$ fand bei Medicare-Bezügern in 306 "U.S. HRRs" nach Kontrolle für soziodemographische Variablen einen positiven Zusammenhang zwischen dem Anteil der Sterbefälle im Spital und der Anzahl Spitalbetten. Fisher et al. ${ }^{16}$ bestätigte diesen Zusammenhang nach zusätzlicher Kontrolle für den Krankheitszustand und für die Ärztedichte. Er zeigte zudem einen positiven Zusammenhang zwischen der Anzahl Spitalbetten und der Anzahl Spitaltage pro Medicare-Bezüger und Jahr. In einer Dissertation aus der Schweiz wurde für Einwohner aus kleinen Gemeinden ohne Spital eine weniger als halb so grosse Wahrscheinlichkeit für den Sterbeort Spital beobachtet als für Einwohner aus kleinen Gemeinden mit Spital ${ }^{22}$. Demgegenüber fanden Gutierrez et al. ${ }^{2}$ in 17 politischen Regionen Spaniens nach Kontrolle für den sozioökonomischen Status sowie für Indikatoren des Bedarfes an Gesundheitsdienstleistungen (z.B. selbst berichteter Gesundheitszustand etc.) keinen Zusammenhang zwischen der Anzahl Spitalbetten und der Inanspruchnahme des Spitals. Sie weisen insbesondere auf die Abhängigkeit der Resultate vom Studiendesign sowie von der Grösse der Regionen hin.

Das Vorhandensein alternativer Versorgungs- und Betreuungsmöglichkeiten (z. B. Alters- oder Pflegeheime, Hospize, Hauspflegedienste) führte in verschiedenen Untersuchungen zu einer vermehrten Inanspruchnahme dieser Angebote sowie zu verminderter Inanspruchnahme des Spitals. Dementsprechend fanden Pritchard et al. ${ }^{6}$ in Regionen mit 
einer hohen Inanspruchnahme der nursing homes sowie mit hohen Gesundheitsausgaben für die Hospize einen tiefen Anteil von Sterbefällen im Spital. Umgekehrt wurde in Kanada von Brownell et al. ${ }^{23}$ im Anschluss an eine Reduktion der Anzahl Spitalbetten in Akutbereich eine Erhöhung der Inanspruchnahme von nursing homes beobachtet. In einer Review von Grande et al. ${ }^{17}$ war die Wahrscheinlichkeit zu Hause zu sterben dann vermindert, wenn ein alternatives stationäres Bettenangebot vorhanden war oder wenn die Betreuung durch eine Hauspflegeorganisation erfolgte, welche an eine stationären Institution angegliedert war.

Gemäss Breyer et al. ${ }^{21}$ wird die Inanspruchnahme einer bestimmten Versorgungsstruktur oder Dienstleistung wesentlich vom Vorhandensein eines alternativen Angebotes sowie von dessen Attraktivität aus Sicht der am Entscheidungsprozess beteiligten Akteure beeinflusst. Im Hinblick auf die Inanspruchnahme des Spitals kommt dabei insbesondere den behandelnden Ärzten eine wichtige Bedeutung zu.

Bezüglich der Ärztedichte pro Einwohner und der Inanspruchnahme des Spitals fanden weder Pritchard et al. ${ }^{6}$ noch Gutierrez et al. ${ }^{2}$ einen signifikanten Zusammenhang. Demgegenüber starben in einer Untersuchung von Polissar et al. ${ }^{15}$ diejenigen Patienten, welche von Spezialisten betreut wurden, eher im Spital als solche, welche von Allgemeinmedizinern behandelt wurden.

Die vorliegende Arbeit geht der Frage nach, welche Bedeutung der stationären und ärztlich-ambulanten medizinischen Versorgung für die Inanspruchnahme der Spitäler durch die ältere Bevölkerung unmittelbar vor dem Tode zukommt. Dazu untersuchen wir für Personen ab 65-jährig in den Jahren 1979 bis 1980 mittels einer ökologischen Analyse den Zusammenhang zwischen den regionalen Anteilen der Sterbefälle im Spital und ausgewählten Indikatoren der regionalen medizinischen Versorgung.

Die folgenden vier Hypothesen sollen dabei auf Ihre Gültigkeit hin untersucht werden: Der regionale Anteil der Sterbefälle im Spital ist umso höher, je grösser das Angebot der Spitäler und der Spezialärzte, je geringer das Angebot der Alters- und Pflegeheime und je geringer das Angebot der Grundversorger in einer Region ist.

\section{Design und Methode}

Als Studien-Design wählten wir einen ökologischen Ansatz (analytical ecological study) ${ }^{24,25}$ mit MS-Regionen als Beobachtungseinheit.

Die Unterteilung der Schweiz nach funktionellen Kriterien in 106 MS-Regionen (mobilité spatiale) wurde $1982 \mathrm{im} \mathrm{Rah-}$ men des NFP 5 "Regionalprobleme" zur Bildung von vergleichbaren und flächendeckenden Analyseeinheiten auf nationaler Ebene vorgenommen. Diese Unterteilung findet heute u.a. im Atlas der Krebsmortalität in der Schweiz ${ }^{26}$, im Strukturatlas der Schweiz ${ }^{27}$ oder im interaktiven Atlas der Schweiz ${ }^{28}$ Verwendung.

Die MS-Regionen setzen sich aus unterschiedlich vielen Gemeinden zusammen. Während die ländlichen Regionen untereinander vergleichbar sind und jeweils einzelnen Einzugsgebieten entsprechen, erstrecken sich in grossstädtischen Räumen die Einzugsgebiete über eine Kernregion in die umgebenden Regionen mit Agglomerationscharakter ${ }^{29}$.

Im analytischen Teil der vorliegenden Untersuchung wurden die fünf Grossstadtzentren mit Universitätsspitälern (Zürich, Basel-Stadt, Bern, Genf, Lausanne) aufgrund der fehlenden Vergleichbarkeit mit anderen Regionen nicht berücksichtigt. Die verbleibenden 101 analysierten MS-Regionen umfassen $75 \%$ der gesamten Bevölkerung ab 65 jährig.

\section{Variablen}

Als Outcome-Variable (abhängige Variable) definierten wir den Anteil der Todesfälle im Spital an den gesamten Todesfällen von Personen ab 65-jährig. Diesen Anteil berechneten wir für jede MS-Region aufgrund der Angaben aus der Sterbefallstatistik des BFS der Jahre 1979 und 1980. Dieses Register enthält für jede in der Schweiz verstorbene Person flächendeckende und systematisch erhobene Angaben über Todesursachen, soziodemographische Informationen sowie bis 1986 auch über den Sterbeort. Letzerer wurde jeweils für die sechs Kategorien "zu Hause", "Spital", "Alters- und Pflegeheim", "im Freien", "Strafanstalt" und "andere oder unbekannt" kodiert.

Zur Überprüfung der Ergebnisse wurde zusätzlich ein indirekt altersstandardisierter "Spital-Sterbefall-Quotient" aus den beobachteten Todesfällen und den aufgrund der Nullhypothese erwarteten Todesfällen berechnet (Nullhypothese: die Anzahl Todesfälle in den einzelnen Regionen unterscheidet sich nicht vom Mittelwert der gesamtschweizerischen Bevölkerung ab 65-jährig).

Aufgrund theoretischer Überlegungen sowie Hinweisen aus der Literatur wählten wir als Haupteinflussvariablen: Anzahl Spitalbetten pro Einwohner ab 18-jährig, Anzahl Alters- und Pflegeheimbetten pro Einwohner ab 65-jährig, Anzahl Konsultationen durch Grundversorger pro Einwohner ab 18-jährig, Anzahl Hausbesuche durch Grundversorger pro Einwohner ab 65-jährig sowie Anzahl Konsultationen 


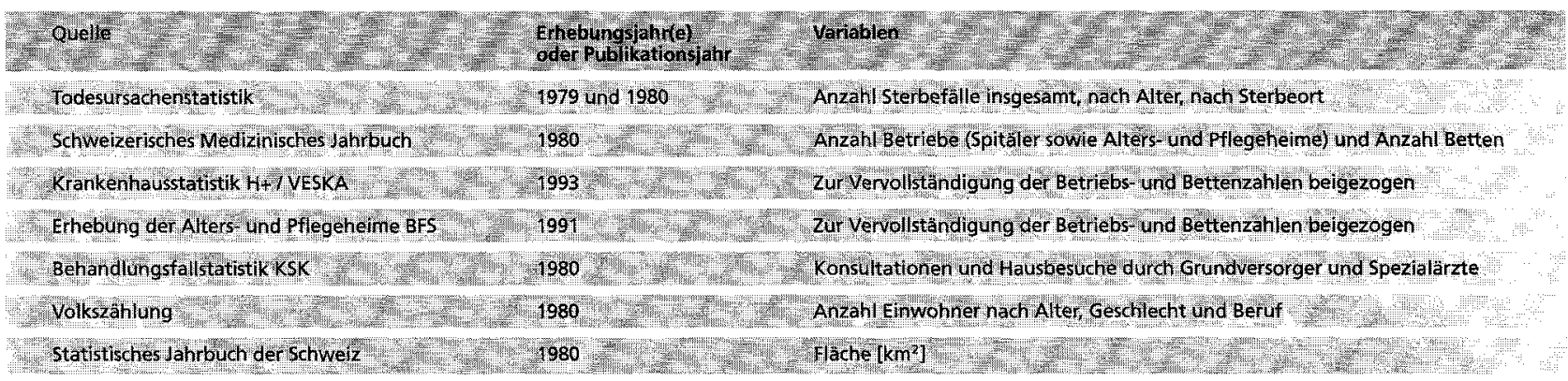

Tabelle 1 Datenquellen

durch Spezialärzte pro Einwohner ab 18-jährig. Als Grundversorger definierten wir Allgemeinmediziner und Allgemeininternisten. Bei den Spitälern wurden alle öffentlichen und privaten Betriebe mit einer Akutabteilung in die Untersuchung eingeschlossen. Institutionen für Ophthalmologie und Pädiatrie/Neonatologie oder Psychiatrie wurden jedoch ausgeschlossen. Bei den Alters- und Pflegeheimen wurden sowohl Betriebe ohne Pflege wie solche mit Pflege (BFS-Typ 1-5) berücksichtigt. Hingegen wurden andere sozialmedizinische Institutionen wie Wohnheime, Institutionen für Invalide, Erholungsheime, Kuranstalten etc. ebenso wie geriatrische Kliniken nicht als Alters- und Pflegeheime gezählt.

Für Alters- und soziostrukturelle Unterschiede zwischen den Regionen kontrollierten wir mit folgenden Variablen: Altersquotient (Anzahl Einwohner ab 65-jährig pro 100 Einwohner 18- bis 64-jährig), Anteil ungelernter Angestellter an der gesamten Bevölkerung, Anteil Hochbetagter (Anzahl Einwohner über 80-jährig an Einwohner ab 65jährig), Bevölkerungsdichte und dominanter Sprachtyp (deutsch oder französich/italienisch).

Die verwendeten Datenquellen sind in Tabelle 1 gezeigt. Wenn immer möglich verwendeten wir Daten aus dem Jahr 1980. Da die Daten über die stationäre Versorgungsstruktur jener Jahre unvollständig sind, mussten zur Vervollständigung neuere Daten beigezogen werden. Alle Daten liegen nach Gemeinde aufgeschlüsselt vor, sodass eine Zuordnung nach MS-Region ohne weiteres möglich ist.

\section{Statistische Methoden}

Zunächst prüften wir die Zusammenhänge der erklärenden Variablen zum Sterbeort Spital in bivariaten Analysen. Die Hypothesen prüften wir mittels einer gewichteten multivariaten linearen Regression. Das optimale Regressionsmodell ermittelten wir mit dem Software Paket von SPSS ${ }^{30}$ durch schrittweises Vorgehen (stepwise procedure) unter
Beibehaltung der Hypothesenvariablen. Als Signifikanzniveau für den Einschluss wurde der P-Wert auf 0,05 und für den Ausschluss auf 0,10 festgesetzt. Als Gewicht setzten wir die gesamte Anzahl der Todesfälle von Personen ab 65jährig ein. Die Voraussetzungen für das Regressionsmodell verifizierten wir graphisch (Residuen-Plots, QQ-Plots). Die Kollinearität wurde ebenfalls überprüft (Variance Inflation Factor, VIF).

Die Robustheit der Ergebnisse testeten wir in mehreren Sensitivitätsanalysen bei verschiedenen Untergruppen und nach Modifikation der Variablen. Dabei verglichen wir vor allem die Regressionskoeffizienten mit den entsprechenden Werten im ursprünglichen Regressionsmodell.

Zur Prüfung einer Abhängigkeit der Ergebnisse vom StadtLand-Charakter der Regionen berechneten wir das Modell für ausschliesslich ländliche Regionen. Die Abhängigkeit vom Sprachtyp testeten wir durch Berechnung der Koeffizienten in den deutschsprachigen Regionen. Weiter prüften wir die Bedeutung der Regionen ohne Spitäler für die Stabilität der Ergebnisse durch Modelltestung in den Regionen mit mindestens einem Spital. Zum Ausschluss einer Abhängigkeit des Modelles von der Begrenzung der Bezugsgrösse für die ärztlichen Konsultationen auf die Einwohner ab 18jährig, testeten wir das Modell zusätzlich, indem wir die Konsultationen auf alle Einwohner ab Geburt bezogen. Zur Überprüfung einer Abhängigkeit der Ergebnisse von den regional unterschiedlichen Altersverteilungen berechneten wir das Regressionsmodell schliesslich noch für den indirekt altersstandardisierten Spital-Sterbefall-Quotienten als abhängige Variable.

\section{Resultate}

\section{Deskriptive Ergebnisse}

In den Jahren 1979 und 1980 verstarben von der älteren Bevölkerung ab 65-jährig insgesamt 89207 Personen. Davon ereigneten sich $54 \%$ der Todesfälle im Spital (m: $55 \%$, 


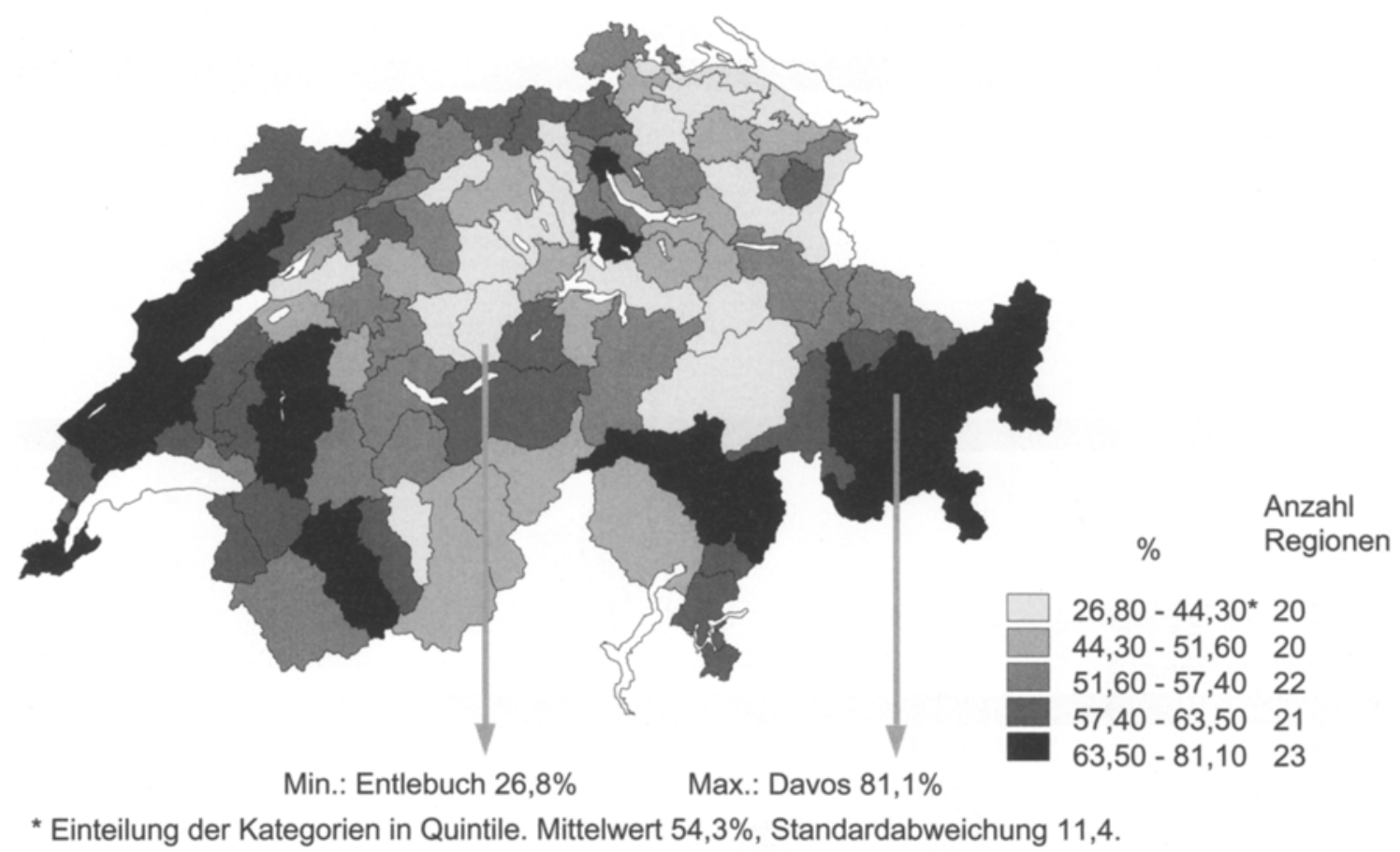

Abbildung 1 Anteile der Todesfälle im Spital von Personen ab 65-jährig in den 106 MS-Regionen der Schweiz in den Jahren 1979 und 1980

f: $53 \%$ ), $29 \%$ im privaten Haushalt (m: $32 \%$, f: $26 \%$ ) und $14 \%$ im Alters- oder Pflegeheim (m: $10 \%$, f: $18 \%$ ). Der Anteil im Spital variierte in den einzelnen MS-Regionen zwischen $27 \%$ und $81 \%$, also um das Dreifache (Abbildung 1). Die vergleichsweise tiefen Anteile von Sterbefällen im Spital befinden sich mehrheitlich in den MS-Regionen der deutschsprachigen Schweiz. In diesem Landesteil erkennt man jedoch auch zahlreiche Regionen mit höheren Anteilen. Demgegenüber weisen alle französischsprachigen, die meisten italienischsprachigen sowie eine von zwei romanischsprachigen Regionen hohe Anteile von Sterbefällen im Spital auf. Ebenfalls hohe Anteile beobachtet man in vier von fünf Grossstadtzentren.

\section{Medizinische Versorgung}

Die einzelnen MS-Regionen unterscheiden sich auch hinsichtlich der medizinischen Versorgung (Tab. 2). In acht deutschsprachigen MS-Regionen gibt es kein Spital und in vier dieser acht Regionen gibt es zudem auch keine Spezialärzte. Die einzige MS-Region ohne Alters- und Pflegeheim entspricht gleichzeitig derjenigen Region mit dem höchsten Anteil Sterbefälle im Spital und der höchsten Anzahl Spezialärzte pro Einwohner ab 18-jährig.

\section{Analytische Ergebnisse}

Die bivariate Analyse der untersuchten Versorgungsvariablen weist einerseits auf einen positiven Zusammenhang der
Spitalbetten und Konsultationen durch Spezialärzte und andererseits auf einen negativen Zusammenhang der Altersund Pflegeheimbetten sowie der Konsultationen und Hausbesuche durch Grundversorger zum Anteil der Sterbefälle im Spital hin.

In der multivariaten Regressionsanalyse wurde ein signifikanter Zusammenhang zwischen dem regionalen Anteil der Sterbefälle im Spital und der Anzahl Konsultationen durch Grundversorger sowie der Anzahl Alters- und Pflegeheimbetten oder der Anzahl Spitalbetten gefunden (Tab. 3).

Die stärkste Assoziation zeigte sich dabei für die Grundversorgerkonsultationen. Eine höhere Anzahl Konsultationen durch Grundversorger war assoziiert mit einem geringeren Anteil Sterbefälle im Spital. Zudem war eine höhere Anzahl Alters- und Pflegeheimbetten sowie einer tieferen Anzahl Spitalbetten mit einem geringeren Anteil Sterbefälle im Spital assoziiert. Entgegen der Hypothese zeigte sich zwischen der Anzahl Hausbesuche und der Anzahl Sterbefälle im Spital ein nicht signifikanter Trend mit mehr Sterbefälle im Spital in Regionen mit einer höheren Anzahl Hausbesuche. Im Weiteren wurde kein signifikanter Zusammenhang zwischen der Anzahl Sterbefälle im Spital und der Anzahl Konsultationen durch Spezialärzte gefunden. Eine zusätzlich durchgeführte Analyse mit alternativen P-Werten für Einund Ausschluss der Variablen $(0,1$ resp. 0,15$)$ ergab keine Veränderung der Resultate. 


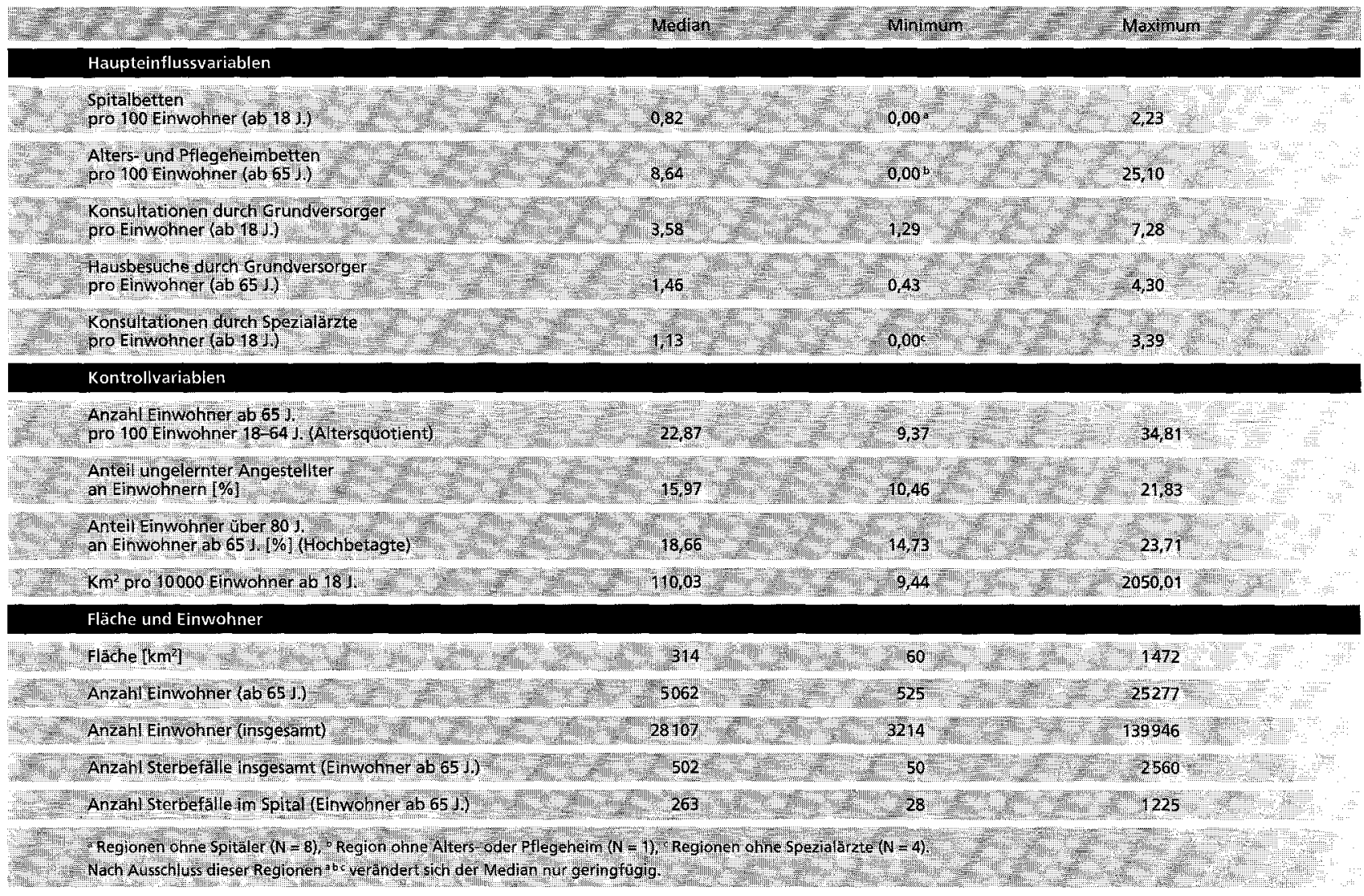

Tabelle 2 Basiswerte für die analysierten 101 MS-Regionen

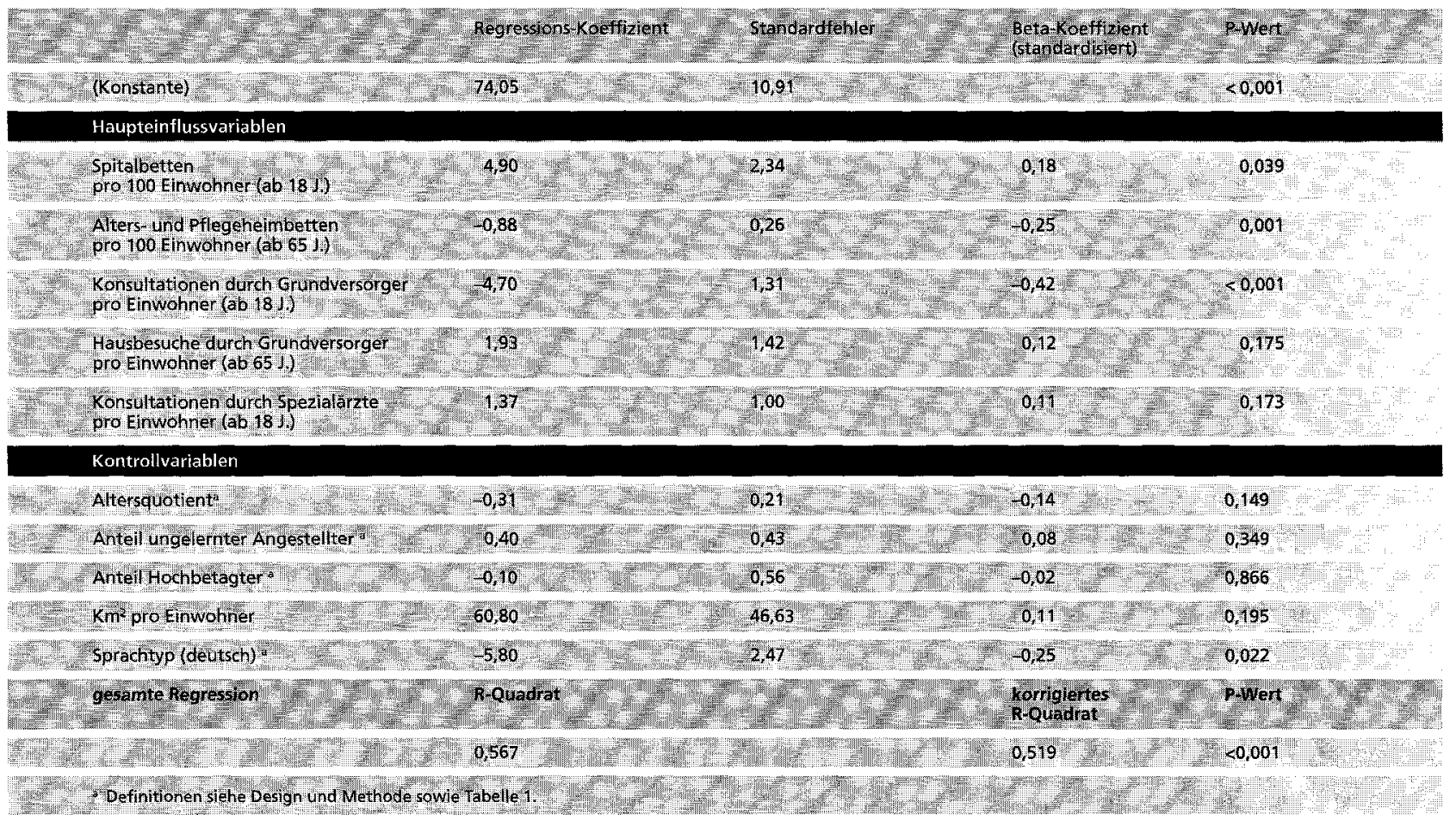

Tabelle 3 Multivariate Zusammenhänge der Haupteinflussvariablen sowie der Kontrollvariablen zum Anteil Sterbefälle im Spital 


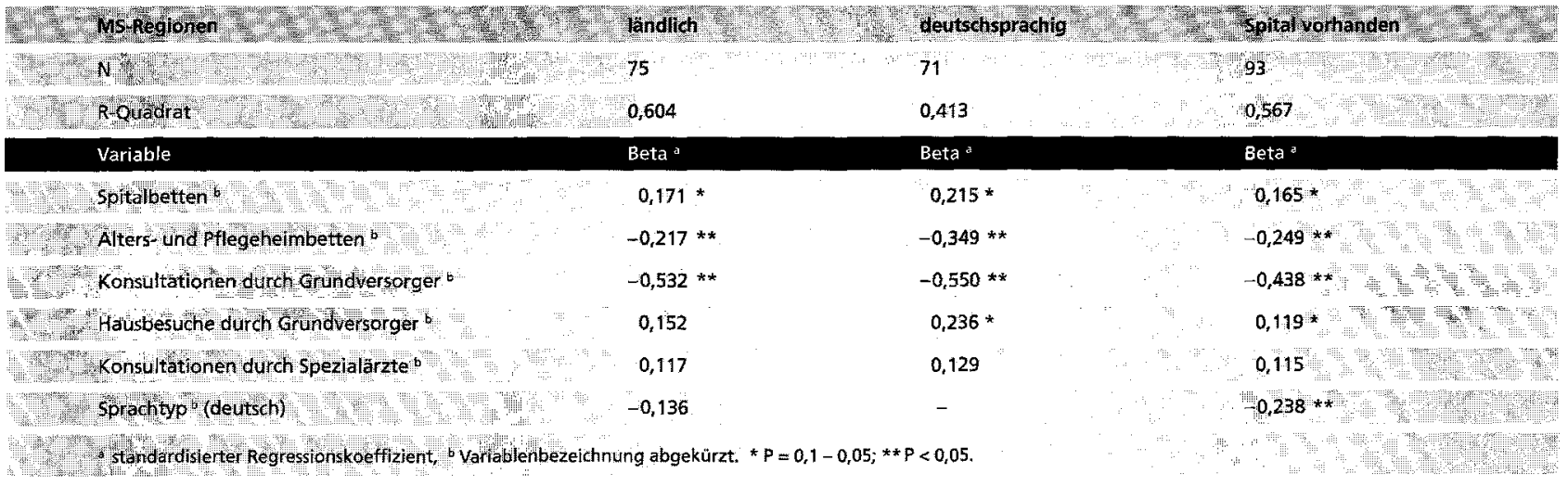

Tabelle 4 Regressionsmodell in ausgewählten Gruppen von MS-Regionen

Bezüglich der Kontrollvariablen zeigt sich einzig für den Sprachtyp eine signifikante Assoziation im Sinne eines tieferen Anteils der Sterbefälle im Spital in den deutschsprachigen Regionen.

Mehr als die Hälfte der Varianz der Anteile der Sterbefälle im Spital wird durch dieses Modell erklärt. Dabei erklärt die Variable der Konsultationen durch Grundversorger alleine $32 \%$ der Varianz.

\section{Sensitivitätsanalysen}

In den Sensitivitätsanalysen erwies sich das Regressionsmodell prinzipiell als robust und zeigte keine grundsätzlichen Veränderung der Koeffizienten oder der Signifikanz der Modelle (Tab. 4). Allerdings erwies sich in den einzelnen Untergruppen der Zusammenhang zwischen der Anzahl Sterbefälle im Spital und den Spitalbetten als nicht mehr signifikant. Diese Veränderung ist möglicherweise auf die geringere Anzahl Beobachtungen zurückzuführen.

\section{Diskussion}

\section{Hauptergebnisse}

In den Jahren 1979 bis 1980 variierten in den einzelnen MSRegionen bei Personen ab 65-jährig die Anteile der Sterbefälle im Spital zwischen $27 \%$ und $81 \%$. Nach Kontrolle für soziodemographische und kulturelle Variablen konnte mehr als die Hälfte dieser Variabilität durch drei Versorgungsvariablen erklärt werden. Die inhaltliche Diskussion der erklärenden Variablen muss aus dreierlei Gründen mit grosser Vorsicht erfolgen.

Erstens darf in einer ökologischen Analyse nicht direkt von den beobachteten Zusammenhängen auf eine Kausalität zwischen den untersuchten Variablen geschlossen werden. Zweitens konnten erklärende Variablen zum Teil nicht zum richtigen Zeitpunkt gemessen werden. Drittens fehlen Variablen, welche aus theoretischen Gründen einbezogen werden sollten, z.B. Daten zur häuslichen Krankenpflege (Spitex).

Die anfänglich formulierten Hypothesen konnten durch die Regressionsanalyse nur teilweise bestätigt werden. Dementsprechend ist der regionale Anteil der Sterbefälle im Spital umso grösser, je geringer die ambulante Konsultationstätigkeit der Grundversorger, je geringer das Angebot der Alters- und Pflegeheime und je grösser das Angebot der Spitäler ist. Dabei erwiesen sich die Konsultationen durch Grundversorger vor den Alters- oder Pflegeheimbetten und den Spitalbetten als stärkste Prädiktoren für den regionalen Anteil der Sterbefälle im Spital.

Die Anzahl Hausbesuche durch Grundversorger sowie die Anzahl Konsultationen durch Spezialärzte blieben ohne signifikante Assoziation zum Sterbeort Spital. Als Erklärung für den Trend von mehr Spitalsterbefällen in Regionen mit mehr Hausbesuchen lässt sich eine häufigere Hospitalisation aufgrund von früher erkanntem schlechtem Gesundheitszustand diskutieren.

Die Betreuung durch Grundversorger scheint in ländlichen Regionen tendenziell eine wichtigere Bedeutung für die medizinische Versorgung unmittelbar vor dem Tode zu haben, als die stationäre Betreuung im Spital. Diese Vermutung ist vereinbar mit der Vorstellung eines besseren sozialen Netzes in den ländlichen Regionen. Zusätzlich wirkt sich in ländlichen Regionen vermutlich auch die grössere Distanz zu den Spitälern sowie eine höhere Alters- und Pflegeheimdichte aus.

Die Wahrscheinlichkeit im Spital zu versterben war in den deutschsprachigen Regionen im Durchschnitt kleiner als in den lateinischsprachigen Landesteilen. Streckeisen ${ }^{10}$ vermutete in điesem Zusammenhang aufgrund einer stärkeren Förderung der ambulanten Betreuungsmöglichkeiten für 
Betagte sowie den geringeren Ausbau von altersspezifischen Wohnmöglichkeiten in der lateinischsprachigen Schweiz eine längere Verweildauer im privaten Haushalt und dadurch einen späteren aber insgesamt häufigeren Übertritt der Betagten in die stationäre Versorgung des Spitals. Dabei wäre jedoch in der lateinischsprechenden Schweiz auch eine intensivere Betreuung durch Grundversorger zu vermuten. Die Anzahl der Konsultationen durch Grundversorger pro Einwohner ist jedoch in den lateinischsprachigen Regionen deutlich geringer als in der deutschsprachigen Schweiz. Demgegenüber ist die Anzahl Konsultationen durch Spezialärzte in der französischen Schweiz tendenziell höher. Die Vermutung, dass diese intensivere Konsultationstätigkeit der Spezialärzte nicht auf einen höheren Bedarf der Bevölkerung (höhere Morbidität) zurückzuführen ist, kann aufgrund der vorliegenden Untersuchung nicht beantwortet werden. Jedoch ist denkbar, dass die Spezialärzte in der lateinischsprachigen Schweiz vermehrt Aufgaben der Grundversorgung übernehmen und durch ihre "berufliche Nähe" zum Spital eine höhere Hospitalisationswahrscheinlichkeit resultiert.

Im Gegensatz zu den meisten Beobachtungen aus der Literatur erwies sich die ambulante ärztliche Versorgung durch Grundversorger in unserer Untersuchung als wichtigster Prädiktor für die Anzahl Sterbefälle im Spital. Dieser Unterschied zu den ausländischen Untersuchungen kann durch die Verwendung der Anzahl Konsultationen durch Grundversorger anstelle der Anzahl Grundversorger erklärt werden. Damit erfassten wir die effektiv erbrachte Leistung besser.

\section{Einschränkungen}

Die Aussagekraft unserer Analyse wird durch mehrere Einschränkungen vermindert. Dabei steht die Problematik der unvollständigen Datengrundlage im Vordergrund. Verschiedene aufgrund der Literatur sowie aufgrund theoretischer Überlegungen wichtige strukturelle Einflussfaktoren wie die ambulante oder teilstationäre pflegerische Betreuung (Spitex, informelle Pflege, Tagesheime etc.) konnten in unserer Studie nicht berücksichtigt werden. Wir vermuten, dass durch derartige Einflussfaktoren ein zusätzlicher Anteil der regionalen Unterschiede erklärt werden könnte. Aufgrund des gewählten Designs werden zusätzlich wichtige individuelle Erklärungsfaktoren wie Gesundheitszustand oder Versicherungsstatus ausgeschlossen.

Aufgrund der Verwendung von verschiedenen Datenquellen aus unterschiedlichen Jahren entspricht die ermittelte Anzahl Betten in den Spitälern oder in den Alters- und Pflegeheimen nicht genau den effektiven Verhältnissen zum untersuchten Zeitpunkt. Insbesondere der vorwiegend in der deutschen Schweiz politisch geförderte Ausbau des Altersheimbereichs ab Ende der 1970er-Jahre ${ }^{10}$ bewirkte eine Zunahme des Angebotes an Altersheimbetten in den Jahren nach 1980 .

Im Weiteren war die Abgrenzung von Betrieben, Abteilungen oder Betten der Akutversorgung gegenüber solchen aus der Langzeitpflege oder der Altersheime aufgrund von fehlenden einheitlichen Definitionen nicht einfach.

Die MS-Regionen als wirtschaftsgeographische Einheiten stimmen weitgehend, aber nicht vollständig mit den medizinischen Versorgungsregionen überein, was wir anhand des Beispiels der Spitalplanungsregionen des Kantons Bern feststellen konnten. Die grössten Diskrepanzen ergeben sich durch die Versorgung der fünf Universitätsspitäler, weshalb die entsprechenden MS-Regionen nicht in die Analyse einbezogen wurden.

Der überregionale Versorgungsauftrag von Universitätsund Kantonsspitälern konnte damit nicht berücksichtigt werden. Ebenso übernehmen die Alters- und Pflegeheime sowie die (Spezial-)Ärzte der ausgeschlossenen Grossstadtzentren oder die Regionen mit stark ausgebauten Versorgungsstrukturen einen massgeblichen Teil der Versorgung von benachbarten MS-Regionen. Die überregionale Versorgung ist vermutlich bei Spitälern und Spezialärzten von grösserer Bedeutung als für die ambulante Betreuung durch Grundversorger. Dadurch könnte die Bedeutung der Spitäler und Spezialärzte für die Inanspruchnahme der Spitäler in unserer Studie unterschätzt werden.

Schliesslich verweisen wir bezüglich der methodischen Problematik ökologischer Analysen auf die weiterführende Literatur ${ }^{25,31}$.

\section{Schlussfolgerungen und Empfehlungen}

Der Anteil der Todesfälle im Spital schwankt in verschiedenen Regionen der Schweiz im Verhältnis 1 zu 3. Mehr als die Hälfte dieser Varianz kann durch strukturelle Unterschiede im Versorgungsangebot dieser Regionen erklärt werden, obwohl sich diese Versorgungsangebote nur zum Teil präzise feststellen liessen. Die Beobachtung grosser regionaler Unterschiede kann zur Verbesserung und Optimierung der medizinischen Versorgung herangezogen werden, sofern sie auf aktuellen Daten beruht. In diesem Sinne empfehlen wir, statistische Daten zur medizinischen Versorgung kontinuierlich zu erfassen.

Aufgrund der gezeigten Zusammenhänge sollten die Massnahmen zur Reduktion der Inanspruchnahme des Spitals umfassender erfolgen und nicht nur das Angebot an Spitalbetten reduzieren. Es sollte jeweils gleichzeitig geprüft werden, wo sich die Verhältnisse der medizinischen Versorgung auf regionaler Ebene, die ambulante ärztliche Tätigkeit der 
Grundversorger sowie das ambulante und stationäre Angebot der Alterspflege verbessern liessen ${ }^{23}$.

Mit dem Ziel einer Verminderung der Gesundheitsausgaben müssten zusätzlich für die einzelnen Massnahmen KostenNutzen-Analysen durchgeführt und die optimale Kombination der Interventionen ermittelt werden.

Unter Vorbehalt der erwähnten Einschränkungen würde gemäss unserem Modell eine Reduktion der Spitalbetten um $10 \%$ eine Verminderung der Inanspruchnahme des Spitals von $1 \%$ bewirken. Denselben Effekt hätte auch der Ausbau des Angebotes an Alters- und Pflegeheimbetten um 5,5\% oder die Förderung der Betreuung von älteren Patienten in der Grundversorgerpraxis mit einer Zunahme der Konsultationen um 2,5\%. Diese "Sparpotienziale" bedürfen einer Bestätigung durch weitere Untersuchungen mit vollständigen und aktuellen Daten. Für den Ausbau der Gesundheitssystemforschung sollten die in gesundheitsstatistischen Erhebungen gewonnenen Daten auf regionaler Ebe-

Zusammentassung

Fragestellung: Vor dem Hintergrund der Diskussion über Massnahmen zum Abbau von Überkapazitatten im Gesund heitswesen untersuchten wir die Bedeutung von medizinischen Versorgungsstrukturen und Dienstieistungen für die Inanspruchnahme des Spitals unmittelbar vor den Tode.

Methoden: Aufgrund der Angaben zum Sterbeort aus der Ster* befallstatistik ermitteiten wir in einem okologischen Design für jede der 106 MS-Regionen der Schweiz die Anteile der Sterbefalle im Spital von Personen ab 65-jährig in den Jahren 1979 bis 1980.

Ergebnisse: Diese Anteile varierten zwischen $27 \%$ und $81 \%$. Trotz Mängeln in den Daten zur stationären und ambulanten Versorgungsstruktur konnte mittels einer multivariaten Regression mehr als die Hälfte der Varianz erklärt werden. Unsere Ergebnisse zeigen, dass der Anteli von Sterbefällen im 5pin tal umso tiefer ist, je mehr Konsultationen durch Grundversorger durchgefüht wurden, je mehr Alters- oder Pflegeheimbetten und je weniger Spitalbetten in den Regionen vorhanden waren (alle Angaben pro Einwohner gerechnet).

Schlussfolgerungen: In der Versorgungsplanung sollten solche systematischen Zusammenhänge zwischen Angebot und Inan" spruchnahme der stationären sowie der ambulanten Versor. gung berücksichtigt werden. Wir empfehlen, Unterschiede zwischen den Regionen in Angebot und Ergebnis medizinischer Leistungen regelmässig mit den jeweils aktuellsten Da* ten zu vergleichen. ne aggregiert werden können. Die MS-Regionen stellen dafür die zur Zeit am besten geeigneten Raumeinheiten dar. Die Angaben zum Sterbeort erlauben einfache und kostengünstige Untersuchungen zur Inanspruchnahme von medizinischen Versorgungsstrukturen auf regionaler Ebene und sollten daher wieder in die amtliche Sterbefallstatistik aufgenommen werden.

Die Bedeutung und Notwendigkeit einer empirisch abgestützten Entscheidungsfindung in der Gesundheits- und Sozialpolitik sollte bei allen Beteiligten erkannt sowie der interdisziplinäre Dialog gefördert werden. Schliesslich empfehlen wir den Aufbau eines nationalen Gesundheitsinformationssystems nach ausländischem Vorbild 1,3,4,31.

\section{Danksagung}

Wir danken dem Bundesamt für Statistik, Sektion Gesundheit, und dem Konkordat der Schweizerischen Krankenkassen für den Zugang zu den verwendeten Daten.

Résumé

Est-ce que la structure des sérvices médicaux influence la proportion des décès à l'hôpital? Une analyse écologique dans les régions «mobilité spatiale» en Suisse

Objectifs: Dans le contexte de la discussion sur la reduction des surcapacités dans les services de santé nous avons examiné l'influence des structures et des services d'approvisionnement médicaux pour le recours à I hôpital directement avant la mort. Méthodes: Sur la base du certificat de décès où l'endroit de mort est enregistré, nous avons déterminé les proportions de décès lhôpital des personnes dans une analyse écologique pour chacune des 106 régions MS de la Suisse à partir de 65 ans de 1979 à 1980.

Résultats: Ces proportions ont varié entre $27 \%$ et $81 \%$. Malgré la carence de les données en mațère de approvisionnement stationnaire et ambulatoire, plus de la moitié de la variance est expliquée par une analyse de régression miltivarie. Nos résulkats démontrent que la proportion des décès à l'hôpital dans une rê. gion est d'autant plus basseque les consultations ont été exécutées par les médecins de premier recours, que plus de lits de maison de retraite ou le moins les lits d'hopital étaient disponibles dans la région (tout les indicateurs calcule par habitant).

Conclusions: Dans la planification des services de santé de telles associations devraient être prises en considération. Nous recommandons de répéter régulierement des analyses écologiques pour comparer des différences interrégionales entre l'offre et résultat de performances médicales avec des données mises à jour. 


\section{Literaturverzeichnis}

1 Nichols LM. Linking state-level health expenditures and utilization data to identify sources of variation in health service prices, utilization and expenditures. Washington, DC: Assistant Secretary for Planning and Evaluation, Health Division, U.S. Department of Health and Human Services, 1999. (DHHS Contract; No HHS-100-95-0021).

2 Gutierrez-Fisac JL, Fletcher AE. Regional differences in hospital use by adults in Spain. Eur J Public Health 1997; 7:254-60.

3 Gaumer GL, Stavins J. Medicare use in the last ninety days of life. Health Serv Res 1992; 26: 725-42.

4 Perkins BB. Reforming medical delivery systems: economic organization and dynamics of regional planning and managed competition. Soc Sci Med 1999; 48: 241-51.

5 Gilliand P. Das Krankenhauswesen der Schweiz, Statistiken 1936-1978, Zukunftsaussichten. Aarau: Schweizerisches Krankenhausinstitut SKI, 1980.

6 Pritchard RS, Fisher ES, Teno JM, et al. Influence of patient preferences and local health system characteristics on the place of death: SUPPORT Investigators: study to understand prognoses and preferences for risks and outcomes of treatment. J Am Geriatr Soc 1998; 46: 1242-50.

7 Felder $S$, Zweifel $P$. Gesundheits- und sozialpolitische Implikationen des Alterungsprozesses. In: Zweifel P, Felder S, eds. Eine ökonomische Analyse des Alterungsprozesses. Bern: Haupt, 1996: 221-35.

8 Lubitz JD, Riley GF. Trends in Medicare payments in the last year of life. $\mathrm{N}$ Engl $\mathrm{J}$ Med 1993; 328: 1092-6.

9 Amold K. Hospize und die "Hospiz-Idee" in Einrichtungen der Altenhilfe. NDV 2; 1998.

10 Streckeisen U, Roost Vischer $L$, Salis Gross C. Die berufliche Konstruktion des Lebensendes: thanatopraktische Handlungsweisen in explorativer Sicht: Schlussbericht an den Schweizerischen Nationalfonds. Bern: Institut für Soziologie, 1992.
11 Bundesamt für Statistik. Kosten des Gesundheitswesens: Pressemitteilung. Bern: Bundesamt für Statistik, 1999.

12 Sommer JH, Höpflinger F. Wandel der Lebensformen und soziale Sicherheit in der Schweiz. Grüsch: Rüegger, 1989.

13 Schüz J, Weber M, Ochsmann R, Huber C. $\mathrm{Zu}$ Hause versterben - wohin geht der Trend? Mainz: Tumorzentrum RheinlandPfalz, 1996.

14 Reilly $P M$, Patten MP. Terminal care in the home. J R Coll Gen Pract 1981; 31: 531-7.

15 Polissar L, Severson RK, Brown NK. Factors affecting place of death in Washington State, 1968-1981. J Community Health 1987; 12: $40-55$

16 Fisher ES, Wennberg JE, Stukel TA, et al. Associations among hospital capacity, utilization, and mortality of US Medicare beneficiaries, controlling for sociodemographic factors. Health Serv Res 2000; 34 : $1351-62$.

17 Grande GE, Addington-Hall JM, Todd CJ. Place of death and access to home care services: are certain patient groups at a disadvantage? Soc Sci Med 1998; 47: 565-79.

18 Zimber $A$, Schaufele $M$, Weyerer $S$. Changes in homes for the aged and nursing homes: functional impairments and behavioral disorders of residents are increasing. Gesundheitswesen 1998; 60: 239-46.

19 Kemper P, Murtaugh CM. Lifetime use of nursing home care. N Engl J Med 1991; 324: 595-600.

20 Bickel $H$. The last year of life: a representative study of deceased patients: living arrangement, place of death and utilization of health resources. Z Gerontol Geriatr 1998; 31: 193-204.

21 Breyer $F, Z$ weifel P. Gesundheitsökonomie. Berlin; Heidelberg: Springer, 1997.

22 Studer T. Einflussfaktoren auf das Sterben zu Hause [Dissertation]. Bern: Universität Bern, 1996.
23 Brownell MD, Roos NP, Burchill C. Monitoring the impact of hospital downsizing on access to care and quality of care. Med Care 1999; 37(6 Suppl): JS135-50.

24 Szklo $M$, Nieto FJ. Ecologic studies. In: Epidemiology beyond the basics. Gaithersburg, Maryland: Aspen Publishers Inc, 2000: 17-23.

25 Morgenstern H. Ecologic study. In: Armitage P, Colton T, ed. Encyclopedia of biostatistics. West Sussex: J. Wiley \& Sons, 1998: 1255-73.

26 Schüler $G$, Bopp $M$. Atlas der Krebsmortalität in der Schweiz. Basel: Birkhäuser, 1997

27 Schuler $M$, Bopp $M$, Brassel KE, Brugger $E A$. Strukturatlas der Schweiz. Zürich: Ex Libris Verlag, 1985.

28 Atlas der Schweiz [Compact Disc]. Bern: Bundesamt für Landestopographie, 2000.

29 Schuler M. Die MS-Regionen. In: Die Raumgliederung der Schweiz: Statistik der Schweiz, Eidgenössische Volkszählung 1990. Bern: Bundesamt für Statistik, 1997: 91-4.

30 SPSS für Windows Release 9.0.1. Chicago: SPSS Inc., 1999.

31 Black $C$, Roos NP, Fransoo R, Martens $P$. Comparative indicators of population health and health care use for Manitoba's Regional Health Authorities: a Populis Project. Manitoba: Manitoba Centre for Health Policy and Evaluation, 1999.

\section{Korrespondenzadresse \\ Dr. med. Christoph Junker Institut für Sozial- und Präventivmedizin der Universität Bern, \\ Finkenhubelweg 11 CH-3012 Bern}

e-mail: junker@ispm.unibe.ch 